\title{
Fitness Cost of
}

\section{Daptomycin-Resistant}

\section{Staphylococcus aureus Obtained from in Vitro Daptomycin Selection Pressure}

\author{
Shuguang Lit, Yuyao Yin', Hongbin Chen, Qi Wang, Xiaojuan Wang and Hui Wang* \\ Department of Clinical Laboratory, Peking University People's Hospital, Beijing, China
}

Daptomycin-resistant (DAP-R) Staphylococcus aureus strains are well documented, but have not been reported in China. To elucidate the evolution adaptability and fitness cost

OPEN ACCESS

Edited by:

Dongsheng Zhou,

Beijing Institute of Microbiology and Epidemiology, China

Reviewed by:

Xiancai Rao,

Third Military Medical University,

China

$\operatorname{Min} \mathrm{Li}$,

Renji Hospital, China

*Correspondence:

Hui Wang

wanghui@pkuph.edu.cn;

whuibj@163.com

tThese authors have contributed equally to this work.

Specialty section:

This article was submitted to

Antimicrobials, Resistance

and Chemotherapy,

a section of the journal

Frontiers in Microbiology

Received: 13 September 2017

Accepted: 26 October 2017

Published: 09 November 2017

Citation:

Li S, Yin Y, Chen $H$, Wang $Q$,

Wang $X$ and Wang $H$ (2017) Fitness

Cost of Daptomycin-Resistant Staphylococcus aureus Obtained from in Vitro Daptomycin Selection Pressure. Front. Microbiol. 8:2199. doi: 10.3389/fmicb.2017.02199 of DAP-R S. aureus, three DAP susceptible strains, Pre3 (MRSA, ST239-t037), Pre5 (MRSA, ST239-t037), and Pre14b (MSSA, ST188-t189), were isolated from patients with bloodstream infections, and serially passaged in Mueller-Hinton broth with a gradient of DAP concentration to select for resistance. Highly DAP-R mutants were obtained after screening for 34 passages. The DAP minimum inhibitory concentrations increased from $0.5 \mu \mathrm{g} / \mathrm{ml}$ in the parent strains to $16 \mu \mathrm{g} / \mathrm{ml}$ in the mutants, which remained tolerant to $4 \mu \mathrm{g} / \mathrm{ml}$ of DAP for more than 160 generations. The growth of the three mutant strains was slower than that of the parent strains, with relative fitness cost of $34.8 \%, 19.2 \%$, and $15.0 \%$, respectively. The in vitro serum tolerance of the mutants was decreased, and the lethality and pathogenicity in mice were weakened $(P<0.01)$. Transmission electron microscopy found that the cell walls of the mutants were significantly thicker (from 38.6\% to $75.4 \%$ ) than those of the parent cells. Mutation L826F of mprF was found in Post14b, G299V, and L473I of mprF and Y225N of walk were found in Post3, while T345A of mprF, S52N of graS, and F473I of walK were found in Post5. Thus, stable DAP-R mutants could be obtained from a middle-short term of in vitro DAP selection, and according to their fitness cost, some prevention and control work may be done to cope with DAP-R S. aureus that may appear in China in the future.

Keywords: in vitro resistance, mutation, daptomycin, Staphylococcus aureus, fitness cost

\section{INTRODUCTION}

Staphylococcus aureus is a frequent cause of many hospital- and community-acquired infections of the skin and soft tissues, pneumonia, bacteremia, endocarditis, and sepsis, with substantial morbidity and mortality. The mortality of S. aureus bacteremia can be as high as 15-25\% (Vogel et al., 2016). Infections due to $S$. aureus with heterogeneous intermediate resistance to vancomycin (hVISA/VISA) potentially fail vancomycin treatment, and daptomycin (DAP) is an important alternative treatment of methicillin-resistant S. aureus (MRSA) (Gould et al., 2013; Miller et al., 2016). 
Daptomycin is a lipopeptide antibiotic produced by Streptomyces roseosporus, and was approved by the Food and Drug Administration (FDA) for soft-tissue infections in 2003 and for $S$. aureus bacteremia and right-sided endocarditis in 2006. DAP acts by calcium- and anionic phospholipid phosphatidylglycerol (PG)-mediated binding to the cell membrane, not the cell wall, of Gram-positive bacteria (Straus and Hancock, 2006). DAP exerts its bactericidal action against $S$. aureus in the stationary growth phase by inhibiting active metabolism without lysis of the target cell (Mascio et al., 2007; Cotroneo et al., 2008).

Daptomycin-resistant (DAP-R) S. aureus strains are well documented and of great concern when treating serious infections caused by this organism (Bayer et al., 2013; Miller et al., 2016). DAP resistance is associated with cell surface charge $(m p r F)$, regulation of dltABCD transcription, and global changes in the expression of VraSR and WalKR (YycFG), two genes that regulate cell membrane stress and maintenance (Bayer et al., 2013; Miller et al., 2016). Agr-defective mutants enhance S. aureus survival during DAP treatment (Pader et al., 2016). As is reported, in vivo DAP-R S. aureus arose during glycopeptide therapy, and the emergence of DAP-R isolates was preceded by a stable VISA or hVISA phenotype (Capone et al., 2016). However, S. aureus strains with this type of DAP resistance have not been reported in China. To further understand the drug resistance mechanism, and to prevent and cope with DAP-R $S$. aureus that may appear in China in the future, two MRSA strains (ST239-t037) and one methicillin-susceptible S. aureus (MSSA) (ST188-t189) from the major genotypes of bacteremia in China were selected (He et al., 2013), then highly DAP$\mathrm{R}$ strains were generated in vitro, and their adaptations were studied.

\section{MATERIALS AND METHODS}

\section{Bacterial Strains}

Staphylococcus aureus strains were isolated from hospitalized patients with bloodstream infections. These wild-type stains were named Pre3 (MRSA, ST239-t037), Pre5 (MRSA, ST239t037), and Pre14b (MSSA, ST188-t189). All were sensitive to DAP, with minimum inhibitory concentrations (MICs) of $0.5 \mu \mathrm{g} / \mathrm{ml}$.

\section{Experimental Selection of DAP-R S. aureus}

Selection of DAP-R strains was conducted in 96-well microtiter plates with $200 \mu$ l Mueller-Hinton $(\mathrm{MH})$ broth per well as previously described (Kim et al., 2014). The DAP gradient concentrations were $0,0.25,0.5,1,2,4,8$, and $16 \mu \mathrm{g} / \mathrm{ml}$ with a calcium ion concentration of $50 \mu \mathrm{g} / \mathrm{ml}$. The plates were incubated at $37^{\circ} \mathrm{C}$ for $20 \mathrm{~h}$ with agitation at $200 \mathrm{rpm}$. Culture supernatants with bacteria growing in the highest DAP concentrations were aspirated and continuously passaged in new DAP gradients until only DAP-R strains with MICs of not less than $8 \mu \mathrm{g} / \mathrm{ml}$ were obtained.

\section{Mutant Stability}

The stability of the DAP-R-mutant strains was confirmed in serial cultures. After $24 \mathrm{~h}$ of culture in $20 \mathrm{ml}$ Luria-Bertani (LB) broth at $35^{\circ} \mathrm{C}$ with agitation at $200 \mathrm{rpm}, 200 \mu \mathrm{l}$ of culture supernatant was removed; $100 \mu \mathrm{l}$ was cultured in fresh LB broth, and $100 \mu \mathrm{l}$ was plated on LB agar. After overnight culture, 100 colonies were randomly selected from the plates, and inoculated on $\mathrm{MH}$ agar plates with $4 \mu \mathrm{g} / \mathrm{ml} \mathrm{DAP}$ and $50 \mu \mathrm{g} / \mathrm{ml}$ calcium ion in a $10 \times 10$ matrix (Wang et al., 2003; Dmowski et al., 2006).

\section{Growth Assay and Calculation of Generation Times}

Wild-type and mutant strains were cultured overnight in LB broth, diluted to an $\mathrm{OD}_{600}$ of 0.01 and grown at $37^{\circ} \mathrm{C}$ with agitation at $200 \mathrm{rpm}$. The cell density was determined every $0.5 \mathrm{~h}$ by measuring the $\mathrm{OD}_{600}$. Both wild-type and mutant $S$. aureus strains were cultured to logarithmic growth phase $\left(\mathrm{OD}_{600}=0.3\right)$ in $\mathrm{LB}$ broth at $37^{\circ} \mathrm{C}$ with agitation at $200 \mathrm{rpm}$. The number of colonies were counted at the beginning and after $1 \mathrm{~h}$ of time interval. Then the generation time was calculated as

$$
G=\frac{t}{3.3 \times \lg \frac{b}{B}},
$$

where $G=$ generation time, $t=$ time interval, $b=$ number of bacteria at the end of the time interval, and $B=$ number of bacteria at the beginning of a time interval (Smits and Riemann, 1988).

\section{Drug Susceptibility Assay}

The MICs of antibiotics in routine clinical use were determined in a central laboratory by broth microdilution following the CLSI M100-S26 guidelines (Clinical and Laboratory Standards Institute [CLSI], 2016).

\section{Fitness Measurements}

DAP-sensitive (DAP-S) wild-type and the corresponding DAP-R strains were diluted to $0.5 \times 10^{7}$ colony-forming units $(\mathrm{CFU}) / \mathrm{ml}$, equal volumes were combined, thus the initial ratio of the strainpairs was infinitely close to $1: 1$, then $10 \mu \mathrm{l}$ of the mixture was added to $20 \mathrm{ml} \mathrm{LB}$ broth and cultured at $35^{\circ} \mathrm{C}$ with agitation at $200 \mathrm{rpm}$. At 24-h intervals, $10 \mu \mathrm{l}$ bacterial subcultures were transferred to fresh LB broth; meanwhile, $10 \mu \mathrm{l}$ was inoculated on drug-free $\mathrm{MH}$ agar, and $10 \mu \mathrm{l}$ on $\mathrm{MH}$ agar containing $1 \mu \mathrm{g} / \mathrm{ml}$ DAP. The number of DAP-S and DAP-R colonies were counted, and after 5 days, adaptive difference was calculated as

$$
S=\ln \left[\left(\frac{\frac{r_{\mathrm{t}}}{s_{\mathrm{t}}}}{\frac{r_{\mathrm{t}-1}}{s_{\mathrm{t}-1}}}\right)^{\frac{1}{17}}\right],
$$

relative adaptive fitness as

$$
F=1+S
$$

and the fitness cost as

$$
C=(1-F) \times 100 \%,
$$


where $r_{t}=$ number of resistant colonies and $s_{t}=$ number of sensitive colonies (Sander et al., 2002; Guo et al., 2012).

\section{Serum Tolerance Assays}

For the in vitro assay of serum tolerance, both wild-type and mutant $S$. aureus strains in logarithmic growth phase $\left(\mathrm{OD}_{600}=0.3\right)$ were diluted to $2 \times 10^{6} \mathrm{CFU} / \mathrm{ml}$ with $0.9 \%$ saline; $50 \mu \mathrm{l}$ of the culture was mixed with $150 \mu \mathrm{l}$ of serum from a healthy person in a $1.5 \mathrm{ml}$ Eppendorf tube and cultured at $37^{\circ} \mathrm{C}$ with agitation at $200 \mathrm{rpm}$. At $0,60,120$, and $180 \mathrm{~min}, 10 \mu \mathrm{l}$ of the mixture was aspirated, combined with $90 \mu \mathrm{l} \mathrm{MH}$ broth, and plated on $\mathrm{MH}$ agar. Bacterial colonies were counted after overnight incubation; each strain was tested three times (Yeh et al., 2012). For the in vivo assay of serum tolerance, wild-type and the corresponding mutant $S$. aureus strains in logarithmic growth phase were diluted to $1 \times 10^{7} \mathrm{CFU} / \mathrm{ml}, 50 \mu \mathrm{l}$ of each culture was mixed and then inoculated into the tail vein of female Balb/c mice. After $72 \mathrm{~h}$, the percentages of DAP-S and DAP$\mathrm{R}$ bacteria in the blood and liver were measured. The animal experiments were approved by the research ethics board at Peking University People's Hospital.

\section{Mouse Pathogenicity and Lethal Ability Assay}

DAP-S and DAP-R strains and a S. aureus 8325-4 (a highvirulent strain) (Novick, 1967; Liu et al., 2010) control strain were incubated at $37^{\circ} \mathrm{C}$ with agitation at $200 \mathrm{rpm}$ overnight and then adjusted to a concentration of $5 \times 10^{9} \mathrm{CFU} / \mathrm{ml}$ with $1 \times \mathrm{PBS}$. Then $100 \mu \mathrm{l}$ volumes of bacterial culture were injected into the tail veins of 15 female BALB/c mice at 8-10 weeks of age. Seven control mice were injected with $100 \mu \mathrm{l}$ of $1 \times$ PBS. The baseline characteristics of the mice in each group did not differ. The mice were monitored for 15 days, every $3 \mathrm{~h}$ for the first 3 days and every $12 \mathrm{~h}$ for the next 12 days (Li et al., 2009).

\section{Phenotypic Analysis}

Cell wall thickness was determined by transmission electron microscopy (FEI Tecnai Spirit). The fluorescence signals of cells were recorded with an EMCCD camera (Photometrics Evolve 512), and the signal intensity was used as a measure of cell membrane potential (Guo et al., 2015; Prindle et al., 2015).

\section{Sequencing of Suspected Genes Probably Associated with Daptomycin Resistance}

Genomic DNA was isolated from both wild-type and mutant S. aureus strains using DNA Extraction Kit (TIANGEN Biotech, China). PCR amplification of mprF, graRS, walKR, dltABCD, and agr $A B C D$ was performed using the primers showed in Supplementary Table S1. The nucleotide sequence data are available at GenBank under the following accession numbers MG029205-MG029258 and MG242038-MG242061.

\section{Statistical Analysis}

Statistical analysis of growth rate was performed with the software GraphPad Prism version 5 using one-way analysis of variance (ANOVA). Continuous variables were statistically analyzed with independent $t$-test. $P<0.05$ was considered to be statistically significant.

\section{RESULTS}

\section{DAP-R S. aureus Mutants}

Highly resistant Post14b, Post3, and Post5 mutants with MICs $=16 \mu \mathrm{g} / \mathrm{ml}$ were generated by continuous passage of susceptible S. aureus Pre14b, Pre3, and Pre5. Bacteria liquids after serial passage were collected and coated on LB agar separately, the morphology of the growing strains in a plate was quite the same, so we randomly picked single colony and did the following study. The sensitivity of $S$. aureus to DAP reduced gradually over time, and as shown in Table 1, after 34 days of serial passage, the DAP MICs increased to $16 \mu \mathrm{g} / \mathrm{ml}$. The MICs of vancomycin and teicoplanin also increased; and those of linezolid, tedizolid, and ciprofloxacin decreased. Little change was observed in the MICs of the other tested antibiotics.

\section{Growth Rates of DAP-R Strains}

The growth rates of DAP-R mutant and DAP-S wild-type $S$. aureus strains are shown in Figure 1. The $\mathrm{OD}_{600}$ of the resistant Post14b strain was significantly lower than that of the Pre14b strain throughout the $24 \mathrm{~h}$ period of measurement (one-way ANOVA, $P<0.05)$. At each phase of the growth curve, the growth rates of the Post $14 \mathrm{~b}$ mutant were reduced compared with the Pre $14 \mathrm{~b}$ strain. The $\mathrm{OD}_{600}$ of the resistant Post 3 strain was significantly higher than that of the Pre 3 strain for the first $6 \mathrm{~h}$, but then was lower from 8 to $24 \mathrm{~h}$; considering the entire curve, growth of the Post3 strain was slower than that of the wild-type strain. The $\mathrm{OD}_{600}$ of the Post 5 strain was significantly lower than

TABLE 1 | Activity of daptomycin and other antibiotics against S. aureus wild-type and mutant strains.

\begin{tabular}{lcccccc}
\hline Strains & pre14b & post14b & pre3 & post3 & pre5 & post5 \\
\hline DAP & 0.5 & 16 & 0.5 & 16 & 0.5 & 16 \\
VAN & 1 & 2 & 1 & 4 & 1 & 2 \\
TEC & 0.5 & 1 & 2 & 4 & 1 & 4 \\
OXA & 0.5 & 0.5 & $>256$ & 256 & $>256$ & 256 \\
FOX & 4 & 4 & $>256$ & 256 & $>256$ & $>256$ \\
LNZ & 4 & 0.032 & 2 & 1 & 2 & 1 \\
TZD & 0.5 & 0.25 & 0.25 & 0.125 & 0.5 & 0.125 \\
CIP & 8 & 0.125 & 64 & 16 & $>64$ & 64 \\
LVX & 0.25 & 0.25 & 8 & 8 & 32 & 32 \\
ERY & 0.5 & 0.5 & $>256$ & $>256$ & $>256$ & $>256$ \\
CLI & 64 & 0.06 & $>256$ & $>256$ & $>256$ & 256 \\
GEN & 0.5 & 1 & $>256$ & $>256$ & $>256$ & $>256$ \\
RIF & $\leq 0.016$ & $\leq 0.016$ & $\leq 0.016$ & $\leq 0.016$ & 0.125 & 4 \\
SXT & 2 & 0.125 & 64 & 64 & 32 & 32
\end{tabular}

DAP, daptomycin; VAN, vancomycin; TEC, teicoplanin; OXA, oxacillin; FOX, cefoxitin; LNZ, linezolid; TZD, tedizolid; CIP, ciprofloxacin; LVX, levofloxacin; ERY, erythromycin; CLI, clindamycin; GEN, gentamicin; RIF, rifampin; SXT, trimethoprimsulfamethoxazole. Data are MIC (microgram/milliliter). 

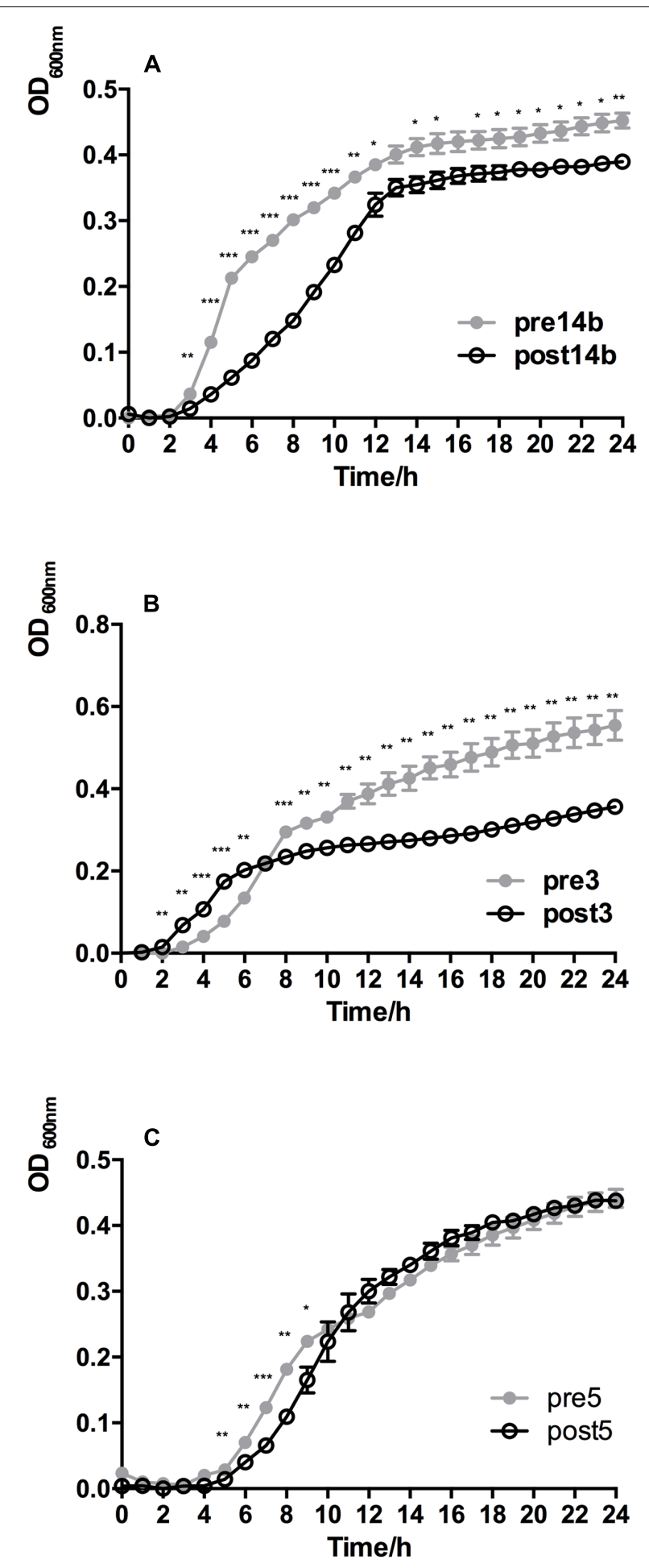

FIGURE 1 | Growth characteristics of DAP-S S. aureus Pre14b, Pre3, and Pre5 strains and their corresponding isogenic derivative Post14b, Post3, and Post5 DAP-R mutants. (A) Pre14b and Post 14b, (B) Pre3 and Post3, and (C) Pre5 and Post5. Strain pairs were grown aerobically in LB liquid culture. The means and standard deviation of three independent experiments are shown. One-way ANOVA was used in statistical analysis. Symbols are defined in the insets. ${ }^{*} P<0.05,{ }^{* *} P<0.01$, and ${ }^{* * *} P<0.001$ for DAP-R vs. DAP-S by analysis of variance. that of the Pre 5 strain from 5 to $9 \mathrm{~h}$ during logarithmic growth, but was not significantly different afterward, indicating similar growth rates.

\section{Stability of Resistance in Mutants}

When grown on $\mathrm{MH}$ agar plates with $4 \mu \mathrm{g} / \mathrm{ml}$ DAP and $50 \mu \mathrm{g} / \mathrm{ml}$ calcium ion, DAP intolerant Post $14 \mathrm{~b}$, Post 3 , and Post 5 colonies were detected on days 7,5 , and 6 , respectively, indicating stable resistance for 4-6 days of culture. Given generation times of $40 \mathrm{~min}$ for Post14b, $27 \mathrm{~min}$ for Post3b, and $45 \mathrm{~min}$ for Post5, resistance would be stable for 210 generations in Post $14 \mathrm{~b}$ and Post $3 \mathrm{~b}$ and 160 generations in Post5.

\section{Fitness Cost of Mutants}

The relative fitness cost, or $C$-values were $34.8 \%$ for Post14b/Pre14b, 19.2\% for Post3/Pre3, and 15.0\% for Post5/Pre5. Thus, the isogenic derivative mutants all had an adaptive cost compared with the parent strains, with the Post14b mutant having the largest fitness cost $(C=34.8 \%)$.

\section{In Vitro and in Vivo Serum Tolerance of Mutants}

The relative numbers of colonies of each strain counted in the in vitro assay of serum tolerance are shown in Supplementary Figure S1. The Post3 and Post14b strains had significantly lower serum tolerance than their wild-type counterparts. The Post5 strain had a lower tolerance for the first $2 \mathrm{~h}$, but an increased tolerance in the third hour. In the assay of in vivo serum tolerance, the wild-type to the mutant strains were all $>1000$ :1, with significant reductions in tolerance. The Pre3/Post3 ratios were 6212:1 in blood and 4174:1 in liver, respectively; the corresponding ratios were 121,266:1 and 1301:1 for Pre5/Post5 and 1192: 1 and 17,274:1 for Pre14b/Post14b in blood and liver, respectively.

\section{Pathogenicity and Lethality of Mutants}

The survival and body weights of surviving mice were shown in Figure 2. The $S$. aureus 8325-4 control strain had the highest lethality; all 15 mice died within $12 \mathrm{~h}$. All 15 mice infected with Pre14b died within $20 \mathrm{~h}$, one mouse (7\%) infected with Post14b died (Figure 2A), four (27\%) infected with Pre3 and 8 (53\%) infected with Pre5 died. No mice infected with Post3 or Post5 dies (Figures 2B,C). The body weights of surviving mice were $17.8 \pm 2.6 \mathrm{~g}$ for Pre3, $20.3 \pm 1.0 \mathrm{~g}$ for Post3, $14.3 \pm 2.0 \mathrm{~g}$ for Pre5, and $20.3 \pm 1.1 \mathrm{~g}$ for Post5. The mice infected with Pre3 and Pre5 were significantly lighter than those infected with their mutant strains $(P<0.05$, Figure 2D). Both the pathogenicity and lethality of the resistant mutant strains were decreased.

\section{Cell Wall Thickness and Cell Membrane Potential}

Cell wall thickness was increased and cell membrane potential was changed in the resistant strains compared with their sensitive counterparts. The cell wall thicknesses were $16.5 \pm 2.3 \mathrm{~nm}$ in Pre14b and $22.9 \pm 2.0 \mathrm{~nm}$ in Post14b, $18.8 \pm 1.9 \mathrm{~nm}$ in Pre 3 and 

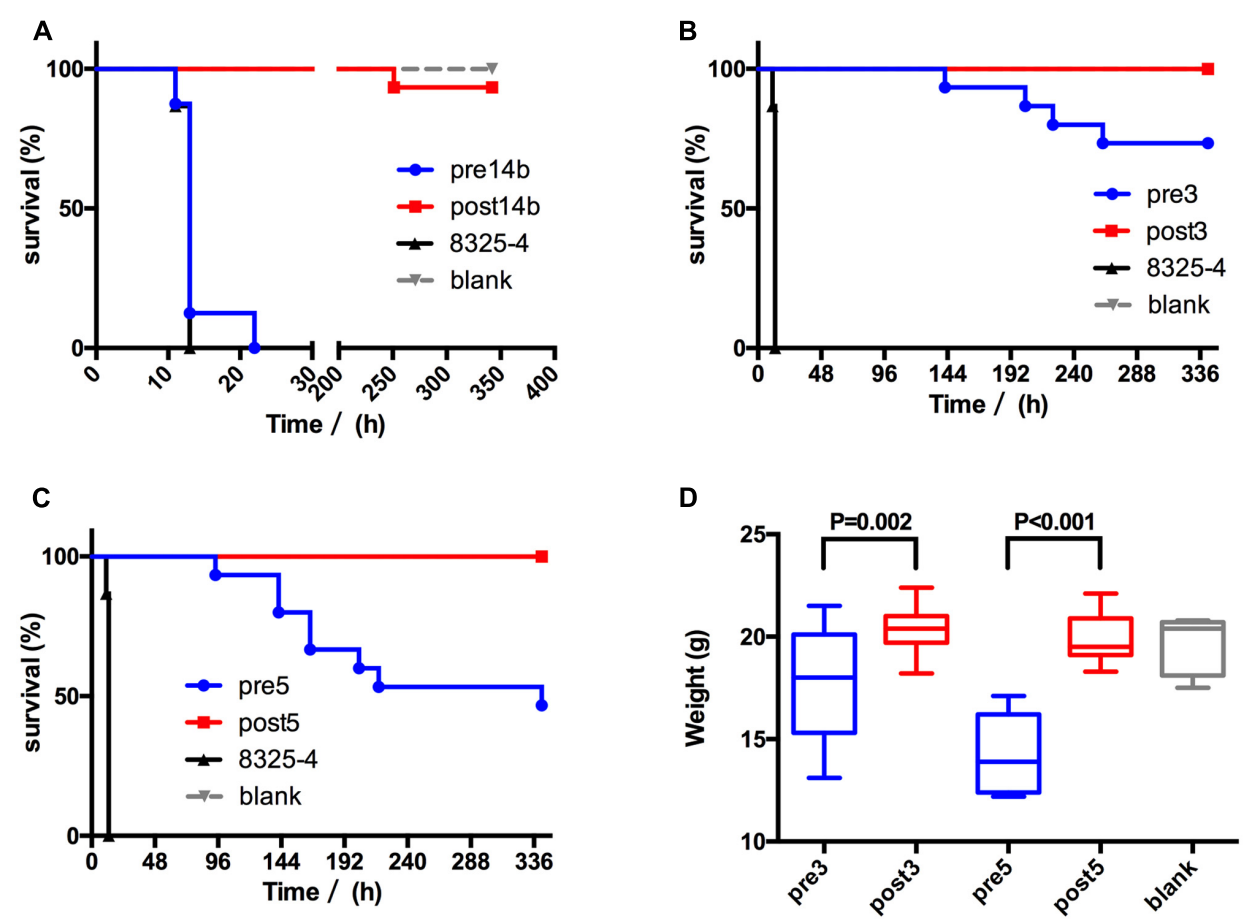

FIGURE 2 | Pathogenicity and lethality ability of S. aureus in mice. The survival curves of (A) Pre14b/Post14b, (B) Pre3/Post3, and (C) Pre5/Post5 are shown. S. aureus 8325-4 was used as high toxicity control, and $1 \times$ PBS was used as blank control. (D) The body weight of surviving mice after infection with Pre3/Post3 and Pre5/Post5. Mice infected by $1 \times$ PBS were used as a blank control.

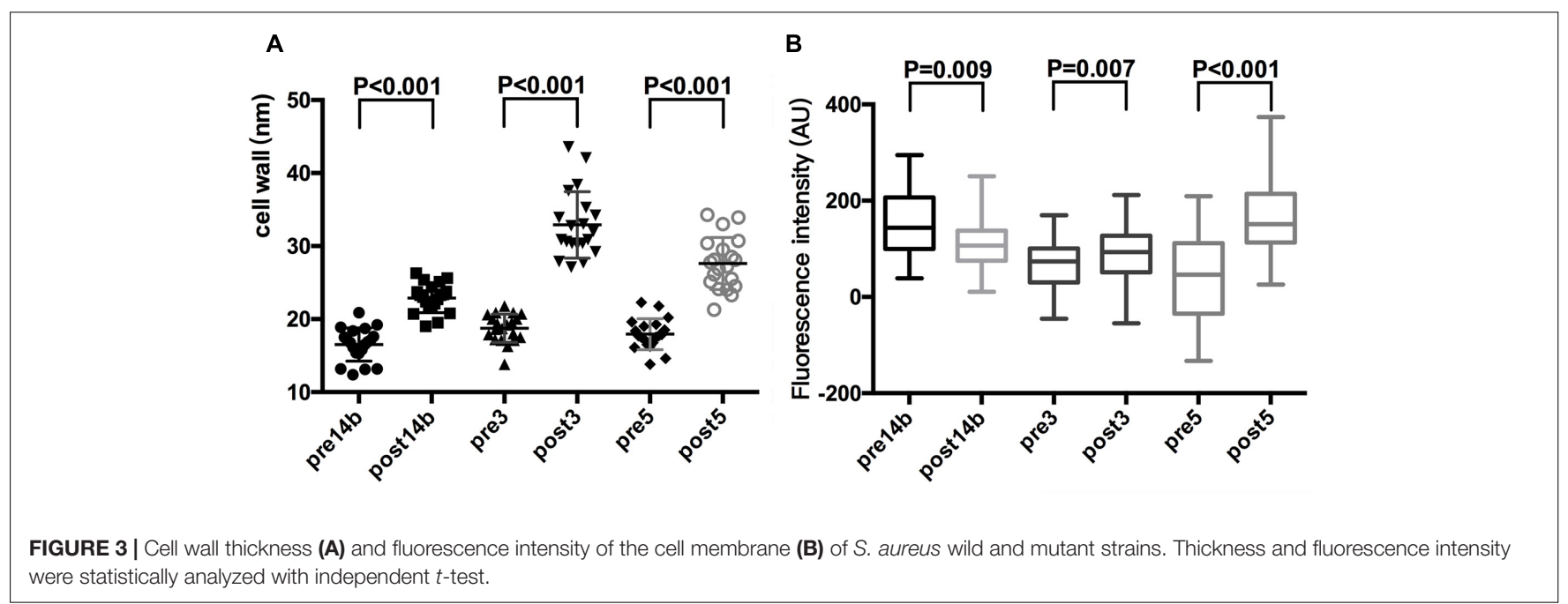

$32.9 \pm 4.5 \mathrm{~nm}$ Post 3 , and $17.9 \pm 2.1 \mathrm{~nm}$ in Pre5 and $27.6 \pm 3.6 \mathrm{~nm}$ in Post5. All increases in thickness were significant $(P<0.001)$, and were $38.6 \%$ in Pre-Post14b, 75.4\% in Pre-Post3b, and 54.0\% in Pre-Post5 (Figure 3A). The fluorescence intensities of the cell membranes were 153.0 \pm 66.0 AU for Pre14b, 63.9 \pm 49.9 AU for Pre3, and 41.8 \pm 88.8 AU for Pre5. The corresponding values for the resistant strains were 109.8 $\pm 48.2 \mathrm{AU}, 93.4 \pm 56.2 \mathrm{AU}$, and $164.2 \pm 78.7$ AU, which were a reduction of $28.3 \%$ in Post $14 \mathrm{~b}$ and increases of $46.1 \%$ in Post 3 and $292.5 \%$ in Post5. All the changes in fluorescence intensity indicated significant changes in cell membrane potentials in the mutant strains $(P<0.01$, Figure 3B).

\section{Detection of Single-Nucleotide Polymorphisms in DAP-R Strains}

The suspected genes may be related with DAP resistance were sequenced, single-nucleotide polymorphisms (SNPs) were found in $m p r F$, graS, and walK, and the mutations of amino acid sequences were shown in Table 2. In detail, for Post14b, mutation was found in L826F of mprF; for Post3, mutation was found in 
TABLE 2 | Amino acid changes in mutant S. aureus strains.

\begin{tabular}{llll}
\hline Strain pairs & mprF & gras & walk \\
\hline Pre14b-Post14b & L826F & No SNP & No SNP \\
Pre3-Post3 & G299V; L473I & No SNP & Y225N \\
Pre5-Post5 & T345A & S52N & F473I
\end{tabular}

Thirteen genes, mprF, graR, graS, walk, walR, dltA, dltB, dltC, dltD, agrA, agrB, agr $C$, and agrD, were sequenced, and mutation only detected in mprF, graS, and walk.

G299V and L473I of mprF, Y225N of walK; for Post5, mutation was found in T345A of mprF, S52N of graS, and F473I of walK. However, no SNPs were found in graR, walR, dltABCD, and $\operatorname{agr} A B C D$.

\section{DISCUSSION}

The benefits of DAP for severe infections caused by multidrugresistant Gram-positive pathogens include bacterial killing without lysing, reduced inflammatory reactions; rapid, concentration-dependent sterilization, a unique mechanism of action without cross-resistance with other antibiotics, and a bactericidal effect on bacterial growth and stability. DAP can also be used as a first-line treatment of life-threatening sepsis or in patients with renal dysfunction in cases with a history of glycopeptide use or a vancomycin MIC > $1.0 \mu \mathrm{g} / \mathrm{ml}$ (Gould et al., 2013; Gonzalez-Ruiz et al., 2016; Miller et al., 2016).

Daptomycin was approved by the American FDA in 2003 and the European EMA in 2006. Cases of clinical treatment failure because of DAP-R strains have been reported (Hsu et al., 2010; Twele et al., 2010), and the emergence of resistance may be associated with high bacterial loads and low doses of DAP ( $\leq 6 \mathrm{mg} / \mathrm{kg} /$ day) in severe infections such as endocarditis. In 2015 and 2016, the State FDA approved the production of DAP by three pharmaceutical companies in China. Consequently, DAP will probably become widely used in China. No clinical DAP$\mathrm{R} S$. aureus isolates have been reported in China, but highly resistant strains with MICs $\geq 16 \mu \mathrm{g} / \mathrm{ml}$ were obtained in vitro by a 34-day DAP induction. As the colonies grown from the passaged bacteria liquid have the same morphology, we presume that in a parallel test may be only one dominant colony could pass down under long time DAP selection; many parallel tests need to do at the same time to get variant resistant strains with different mutations. The MICs of two MRSA strains and the MSSA strain isolated from inpatients with bloodstream infections increased from 0.5 to $16 \mu \mathrm{g} / \mathrm{ml}$ and remained tolerant to $4 \mu \mathrm{g} / \mathrm{ml}$ DAP for more than 160 generations. The results confirmed that $S$. aureus could grow in the presence of high DAP concentrations, and that DAP resistance was stably inherited.

Relatively small increases in the MICs of vancomycin and teicoplanin were detected, no teicoplanin-resistant strains appeared, and only one mutant strain (Post3) had intermediate resistance to vancomycin $(\mathrm{MIC}=4 \mu \mathrm{g} / \mathrm{ml})$. The MIC increase of glycopeptides may be due to mutation in $m p r F$, which changed in all three mutants and correlated with cell wall cross-linking. On the other hand, the MICs of linezolid, tedizolid, and ciprofloxacin decreased slightly. It was not determined whether that resulted in fitness cost or unknown genetic changes that may be revealed after genome sequencing. The MICs of oxacillin, cefoxitin, and the other tested antibiotics changed very little.

The growth rate of mutants was decreased significantly in two strain pairs (Pre14/Post14b and Pre3/Post3), and were similar in Pre5/Post5. The growth rate of the DAP-R mutants was not always slowed when cultured alone with sufficient medium. However, when cultured together with the wild-type strain and adequate nutrition in vitro or inoculated into mice in vivo, the number of mutant colonies was far less than the number of wild-type, and all mutant strains showed fitness cost with $C$-values between $15.0 \%$ and $34.8 \%$. Because of fitness costs, the mutant strains performed weakly in the competitive growth experiments. In vivo, the conditions were more complex than in vitro, the decreased growth of the mutant strains may have resulted from both low competitiveness and low serum tolerance. The reductions of lethality and pathogenicity following infection in mice further confirmed the fitness costs of the DAP-R strains.

The fitness costs demonstrated by $C$-values were associated with changes in cell wall thickness and cell membrane potential. The cell wall thicknesses of all the mutant strains were significantly increased $(P<0.001)$, which is consistent with previous reports of vancomycin non-susceptible strains (Hanaki et al., 1998; Utaida et al., 2003). However, DAP and vancomycin resistance may be caused by different mechanisms, as the bacteria with thickened cell walls may be resistant to only one of the two drugs, such as Post $14 \mathrm{~b}$ and Post5, which had high DAP resistance, but both were sensitive to vancomycin (MIC $\leq 2 \mu \mathrm{g} / \mathrm{ml}$ ).

Staphylococcus aureus cell membrane lipids are mainly negatively charged phospholipids such as PG and cardiolipin. When calcium ions are present, DAP causes cell death by binding to and depolarizing the cell membrane (Stefani et al., 2015). When changes in the composition of the cell membrane result in a decrease in its negative charge and increase in mobility, the resulting decrease in DAP binding leads to resistance. The decrease in cell membrane potential in the Post $14 \mathrm{~b}$ strain was consistent with this cause of DAP resistance. However, Post3 and Post 5 had increased membrane potentials, which might not have favored DAP resistance; other factors that can lead to DAP resistance need to be evaluated.

In this study, all the DAP-R strains had a fitness cost that differed in each pair of DAP-R and DAP-S strains. Genetic mutations were found in mprF, graS, and walK. To our knowledge, G299V and L473I in mprF, S52N in graS, and Y225N and F473I in walK were the novel variations have not been reported. As $m p r F$ is associated with cell surface charge and cell wall cross-linking, WalK regulates cell membrane stress and maintenance, GraS regulates VraFG expression, involved in cationic antimicrobial peptide (CAMP) sensing and signal transduction to promote $S$. aureus resistance (Falord et al., 2012; Bayer et al., 2013; Miller et al., 2016), we presume that genetic changes of these three genes played important roles in DAP resistance. However, as drug resistance is complex property that depends on multigene activity, and the mutant strains not always showed consistent characteristics, other genes beside $m p r F$, graS, and walK may be changed in the passages, thus 
whole-genome sequencing and comparative genome analysis of wild-type, final mutants, and resistant intermediates obtained during serial passage is warranted. What's more, knockout and complementation strains of key genes would be constructed to confirm their roles in DAP resistance.

\section{ETHICS STATEMENT}

The animal experiments were approved by the research ethics board at Peking University People's Hospital, with the ERP No. 81371856 and IACUC Ethics Approval No. 2013-79.

\section{AUTHOR CONTRIBUTIONS}

HW conceived and designed the study. SL, YY, HC, QW, and $\mathrm{XW}$ performed experiments described in this study. SL and YY

\section{REFERENCES}

Bayer, A. S., Schneider, T., and Sahl, H. G. (2013). Mechanisms of daptomycin resistance in Staphylococcus aureus: role of the cell membrane and cell wall. Ann. N. Y. Acad. Sci. 1277, 139-158. doi: 10.1111/j.1749-6632.2012.06819.x

Capone, A., Cafiso, V., Campanile, F., Parisi, G., Mariani, B., Petrosillo, N., et al. (2016). In vivo development of daptomycin resistance in vancomycinsusceptible methicillin-resistant Staphylococcus aureus severe infections previously treated with glycopeptides. Eur. J. Clin. Microbiol. Infect. Dis. 35, 625-631. doi: 10.1007/s10096-016-2581-4

Clinical and Laboratory Standards Institute [CLSI] (2016). Performance Standards for Antimicrobial Susceptibility Testing, 26th Edn. Wayne, PA: CLSI.

Cotroneo, N., Harris, R., Perlmutter, N., Beveridge, T., and Silverman, J. A. (2008). Daptomycin exerts bactericidal activity without lysis of Staphylococcus aureus. Antimicrob. Agents Chemother. 52, 2223-2225. doi: 10.1128/AAC. 01410-07

Dmowski, M., Sitkiewicz, I., and Ceglowski, P. (2006). Characterization of a novel partition system encoded by the delta and omega genes from the streptococcal plasmid pSM19035. J. Bacteriol. 188, 4362-4372. doi: 10.1128/JB. 01922-05

Falord, M., Karimova, G., Hiron, A., and Msadek, T. (2012). GraXSR proteins interact with the $\mathrm{VraFG} \mathrm{ABC}$ transporter to form a five-component system required for cationic antimicrobial peptide sensing and resistance in Staphylococcus aureus. Antimicrob. Agents Chemother. 56, 1047-1058. doi: 10.1128/AAC.05054-11

Gonzalez-Ruiz, A., Seaton, R. A., and Hamed, K. (2016). Daptomycin: an evidencebased review of its role in the treatment of Gram-positive infections. Infect. Drug Resist. 9, 47-58. doi: 10.2147/IDR.S99046

Gould, I. M., Miro, J. M., and Rybak, M. J. (2013). Daptomycin: the role of highdose and combination therapy for Gram-positive infections. Int. J. Antimicrob. Agents 42, 202-210. doi: 10.1016/j.ijantimicag.2013.05.005

Guo, B., Abdelraouf, K., Ledesma, K. R., Nikolaou, M., and Tam, V. H. (2012). Predicting bacterial fitness cost associated with drug resistance. J. Antimicrob. Chemother. 67, 928-932. doi: 10.1093/jac/dkr560

Guo, X., Jin, X., Lv, X., Pu, Y., and Bai, F. (2015). Real-time visualization of perylene nanoclusters in water and their partitioning to graphene surface and macrophage cells. Environ. Sci. Technol. 49, 7926-7933. doi: 10.1021/acs.est. $5 \mathrm{~b} 01880$

Hanaki, H., Kuwahara-Arai, K., Boyle-Vavra, S., Daum, R. S., Labischinski, H., and Hiramatsu, K. (1998). Activated cell-wall synthesis is associated with vancomycin resistance in methicillin-resistant Staphylococcus aureus clinical strains Mu3 and Mu50. J. Antimicrob. Chemother. 42, 199-209. doi: 10.1093/ jac/42.2.199

He, W., Chen, H., Zhao, C., Zhang, F., Li, H., Wang, Q., et al. (2013). Population structure and characterisation of Staphylococcus aureus from bacteraemia at wrote the draft, and HW revised it. All authors approved the final version.

\section{FUNDING}

The study was supported by National Natural Science Foundation of China (Grant Nos. 81371856, 81501775, and 81625014).

\section{SUPPLEMENTARY MATERIAL}

The Supplementary Material for this article can be found online at: https://www.frontiersin.org/articles/10.3389/fmicb. 2017.02199/full\#supplementary-material

FIGURE S1 | In vitro serum tolerance of wild-type and mutant strains.

multiple hospitals in China: association between antimicrobial resistance, toxin genes and genotypes. Int. J. Antimicrob. Agents 42, 211-219. doi: 10.1016/j. ijantimicag.2013.04.031

Hsu, L. Y., Leong, M., Balm, M., Chan, D. S., Huggan, P., Tan, T. Y., et al. (2010). Six cases of daptomycin-non-susceptible Staphylococcus aureus bacteraemia in Singapore. J. Med. Microbiol. 59(Pt 12), 1509-1513. doi: 10.1099/jmm.0. 022533-0

Kim, S., Lieberman, T. D., and Kishony, R. (2014). Alternating antibiotic treatments constrain evolutionary paths to multidrug resistance. Proc. Natl. Acad. Sci. U.S.A. 111, 14494-14499. doi: 10.1073/pnas.1409800111

Li, M., Diep, B. A., Villaruz, A. E., Braughton, K. R., Jiang, X., DeLeo, F. R., et al. (2009). Evolution of virulence in epidemic community-associated methicillinresistant Staphylococcus aureus. Proc. Natl. Acad. Sci. U.S.A. 106, 5883-5888. doi: 10.1073/pnas.0900743106

Liu, Y., Wu, N., Dong, J., Gao, Y., Zhang, X., Mu, C., et al. (2010). Hfq is a global regulator that controls the pathogenicity of Staphylococcus aureus. PLOS ONE 5:e13069. doi: 10.1371/journal.pone.0013069

Mascio, C. T., Alder, J. D., and Silverman, J. A. (2007). Bactericidal action of daptomycin against stationary-phase and nondividing Staphylococcus aureus cells. Antimicrob. Agents Chemother. 51, 4255-4260. doi: 10.1128/AAC. 00824-07

Miller, W. R., Bayer, A. S., and Arias, C. A. (2016). Mechanism of action and resistance to daptomycin in Staphylococcus aureus and enterococci. Cold Spring Harb. Perspect. Med. 6:a026997. doi: 10.1101/cshperspect.a026997

Novick, R. (1967). Properties of a cryptic high-frequency transducing phage in Staphylococcus aureus. Virology 33, 155-166. doi: 10.1016/0042-6822(67) 90105-5

Pader, V., Hakim, S., Painter, K. L., Wigneshweraraj, S., Clarke, T. B., and Edwards, A. M. (2016). Staphylococcus aureus inactivates daptomycin by releasing membrane phospholipids. Nat. Microbiol. 2:16194. doi: 10.1038/nmicrobiol. 2016.194

Prindle, A., Liu, J., Asally, M., Ly, S., Garcia-Ojalvo, J., and Suel, G. M. (2015). Ion channels enable electrical communication in bacterial communities. Nature 527, 59-63. doi: 10.1038/nature15709

Sander, P., Springer, B., Prammananan, T., Sturmfels, A., Kappler, M., Pletschette, M., et al. (2002). Fitness cost of chromosomal drug resistanceconferring mutations. Antimicrob. Agents Chemother. 46, 1204-1211. doi: 10.1128/AAC.46.5.1204-1211.2002

Smits, J. D., and Riemann, B. (1988). Calculation of cell production from $\left[{ }^{3} \mathrm{H}\right]$ thymidine incorporation with freshwater bacteria. Appl. Environ. Microbiol. 54, 2213-2219.

Stefani, S., Campanile, F., Santagati, M., Mezzatesta, M. L., Cafiso, V., and Pacini, G. (2015). Insights and clinical perspectives of daptomycin resistance in Staphylococcus aureus: a review of the available evidence. Int. J. Antimicrob. Agents 46, 278-289. doi: 10.1016/j.ijantimicag.2015.05.008 
Straus, S. K., and Hancock, R. E. (2006). Mode of action of the new antibiotic for Gram-positive pathogens daptomycin: comparison with cationic antimicrobial peptides and lipopeptides. Biochim. Biophys. Acta 1758, 1215-1223. doi: 10. 1016/j.bbamem.2006.02.009

Twele, L., Moyen, E., Zhang, K., Dalton, B., Church, D., and Conly, J. (2010). Methicillin-resistant Staphylococcus aureus endocarditis and de novo development of daptomycin resistance during therapy. Can. J. Infect. Dis. Med. Microbiol. 21, 89-93.

Utaida, S., Dunman, P. M., Macapagal, D., Murphy, E., Projan, S. J., Singh, V. K., et al. (2003). Genome-wide transcriptional profiling of the response of Staphylococcus aureus to cell-wall-active antibiotics reveals a cell-wall-stress stimulon. Microbiology 149(Pt 10), 2719-2732. doi: 10.1099/mic.0.26426-0

Vogel, M., Schmitz, R. P., Hagel, S., Pletz, M. W., Gagelmann, N., Scherag, A., et al. (2016). Infectious disease consultation for Staphylococcus aureus bacteremia a systematic review and meta-analysis. J. Infect. 72, 19-28. doi: 10.1016/j.jinf. 2015.09.037

Wang, M., Tran, J. H., Jacoby, G. A., Zhang, Y., Wang, F., and Hooper, D. C. (2003). Plasmid-mediated quinolone resistance in clinical isolates of Escherichia coli from Shanghai, China. Antimicrob. Agents Chemother. 47, 2242-2248. doi: 10.1128/AAC.47.7.2242-2248.2003

Yeh, F. C., Yeh, K. M., Siu, L. K., Fung, C. P., Yang, Y. S., Lin, J. C., et al. (2012). Increasing opsonizing and killing effect of serum from patients with recurrent K1 Klebsiella pneumoniae liver abscess. J. Microbiol. Immunol. Infect. 45, 141-146. doi: 10.1016/j.jmii.2011. 12.006

Conflict of Interest Statement: The authors declare that the research was conducted in the absence of any commercial or financial relationships that could be construed as a potential conflict of interest.

Copyright (c) 2017 Li, Yin, Chen, Wang, Wang and Wang. This is an open-access article distributed under the terms of the Creative Commons Attribution License (CC BY). The use, distribution or reproduction in other forums is permitted, provided the original author(s) or licensor are credited and that the original publication in this journal is cited, in accordance with accepted academic practice. No use, distribution or reproduction is permitted which does not comply with these terms. 